\title{
Hippocampal lesions during rearing and transfer of perceptual learning in rats*
}

\author{
BARRY S. ANTON $\dagger$ \\ University of Puget Sound, Tacoma, Washington 98416 \\ and \\ THOMAS L. BENNETT \\ Colorado State University, Fort Collins, Colorado 80521
}

\begin{abstract}
Bilateral lesions of the hippocampus in humans result in severe deficits of recent memory which consist of an inability to recall events a few minutes after they have occurred. On the other hand, such patients demonstrate normal abilities to recall events that transpired before the operation. Studies employing animals as subjects have produced contrary evidence concerning a memory-consolidation view of hippocampal functioning because hippocampal animals are not deficient in learning a variety of tasks. In the present research, the recent memory hypothesis was reexamined employing the early experience paradigm of Gibson and Walk (1956). This methodological variation provided a testing situation more analogous to the human testing situation in that animals were required to utilize salient cues from their environment and to transfer this information to a subsequent discrimination task. It was found that animals receiving bilateral hippocampectomy before exposure to the to-be-discriminated stimuli fared no better in a subsequent discrimination test than animals not exposed to the stimuli. In addition, animals undergoing hippocampal ablation after exposure to the forms demonstrated significantly better performance in the test situation than nonexposed animals. The findings of the present research provide some support for the recent memory hypothesis of hippocampal functioning.
\end{abstract}

Recent interest in the hippocampus and its possible functions in associative processes was first generated by human clinical observations which indicated that bilateral damage to this structure resulted in severe deficits in recent memory, that is, in the ability to add new information to long-term memory. These effects seemed to be specific to the short-term memory mechanism, because general intelligence and the ability to recall information acquired prior to surgery were generally unaffected. Further, the extent of this deficit was found to vary directly with the amount of bilateral hippocampal involvement produced by the ablation. These observations led Penfield and Milner (1958) to suggest that the initial encoding of memory traces was accomplished by hippocampal mechanisms. For a review of these findings, the reader is referred to Milner (1966).

Attempts to find support for and elucidate the basis of this learning deficit using infrahuman subjects have been discouraging (see Douglas, 1967; Kimble, 1968). While lower animals with hippocampal damage are certainly behaviorally different from normal animals, particularly with regard to their perseverative behavior patterns, the animal-lesion literature has failed to substantiate or refute the recent memory hypothesis of hippocampal functioning. Lower animals can learn a variety of tasks in the absence of an intact hippocampus.

\footnotetext{
*This article is based on a dissertation submitted by the first author in partial fulfillment of the requirements for the Ph.D. degree at Colorado State University.

tRequests for reprints should be sent to: Barry S. Anton, Department of Psychology, University of Puget Sound, Tacoma, Washington, 98416 .
}

It appears plausible that the lack of supporting evidence for the recent memory hypothesis when infrahumans have served as experimental subjects has been the product of methodological differences in the human vs lower animal inquiries. The almost exclusive use of two-choice discrimination problems in the animal-lesion studies may have resulted in hippocampal investigators failing to tap the relevant dimensions necessary to evoke evidence of a recent memory deficit. In the majority of the human studies, the testing situation involved recall of chains of verbal items or complex events from the subject's daily life.

An alternative technique for examining the recent memory hypothesis in rodents, in a condition which is perhaps more analogous to the testing regimen used with the human hippocampal patients, is to require the infrahuman to use information from its past environmental experiences in a transfer paradigm. Specifically, one could examine the effects of hippocampal lesions on transfer of perceptual learning using the early experience model of Gibson and Walk (1956). We have used this paradigm in a number of studies in recent years to examine the influence of early experience on later discrimination learning (e.g., Bennett \& Ellis, 1968; Bennett, Anton, \& Levitt, 1971).

The early experience paradigm follows a procedure in which experimental animals are exposed to the to-be-discriminated forms (e.g., circles and triangles) during rearing. At maturity, the animals are required to learn to discriminate between these stimuli as a test of the early experience conditions. It has generally been 
found that preexposed animals learn the discrimination problem significantly faster than do nonexposed control subjects. This paradigm requires animals to acquire information in their daily living environments and later transfer this information to a discrimination problem. It was hypothesized that the amount transferred to the subsequent discrimination problem by hippocampectomized animals might provide both a sensitive index of the effects of hippocampectomy and a more analogous testing situation to that employed in the human investigations. As a result, the procedure might provide a better framework for assessing the validity of the recent memory hypothesis for infrahuman subjects.

\section{METHOD}

\section{Experimental Design}

Albino rats were reared in a visually sparse environment from birth until the completion of discrimination training. Stimulus placement procedures were begun at 50 days of age for the animals receiving preexposure to the to-be-discriminated forms, and placement procedures were continued for the duration of the experiment. Half of the animals received early experience with the forms and half did not. Within each of the early-experience conditions, the subjects received one of four kinds of surgical treatment: no lesion, cortical control lesion, early hippocampal ablation, or late hippocampal ablation. The cortical control animals were run because aspiration lesions of the hippocampus also destroy the cortex. A detailed description of the resulting groups and the surgical procedures utilized to effectuate the lesions are presented below. At 100 days of age, the subjects were placed on a 23-h food deprivation schedule in preparation for the beginning of discrimination pretraining at 110 days old. Following four sessions of pretraining, the animals of all groups were given a circle-triangle discrimination problem as a test of the effects of the experimental manipulations.

\section{Subjects}

The subjects were male albino rats, with eight animals assigned to each of the eight groups.

\section{Experimental Groups}

The conditions of early exposure and surgical treatment resulted in eight experimental groups as follows: (a) In Group NNE (normal nonexposed), the animals were unoperated and not preexposed to the stimuli. (b) In Group NE (normal exposed), the animals were also unoperated but were preexposed to the to-be-discriminated stimuli. (c) In Group CNE (cortical nonexposed), the animals received bilateral lesions of the neocortex overlying the hippocampus, and experience with the to-be-discriminated forms was not provided. (d) In Group CE (cortical exposed), the animals were identically treated surgically as Group CNE and were preexposed to the stimuli. (e) In Group EHNE (early hippocampal nonexposed), the rats received bilateral aspiration of the hippocampus at age 45 days and no early experience with the shapes. (f) In Group EHE (early hippocampal exposed), the animals also received bilateral aspiration of the hippocampus at 45 days but following 5 days recovery, experience with the to-be-discriminated stimuli was introduced. (g) In Group LHNE (late hippocampal nonexposed), the animals received bilateral destruction of the hippocampus at 105 days of age but no early experience with the forms. (h) In Group LHE (late hippocampal exposed), animals received experience with the to-be-discriminated stimuli from age 50 days, and at age 105 days the hippocampus was bilaterally removed through aspiration. During the course of the experiment, one animal died from the CNE and LHE groups.

\section{Rearing}

The animals were reared (three to five per cage) in Hoeltge small-animal cages, measuring $9 \times 9 \times 15$ in., which were placed in compartments measuring $2 \mathrm{ft}$ square. The sides, bottoms, and tops of the compartments were painted flat white. The opening of each compartment was two feet from the white wall of the room in which the animals were housed. Food and water were available ad lib. Each of the compartments was illuminated from 7:00 a.m. to $6: 00$ p.m. daily by a $25-\mathrm{W}$ bulb attached to the compartment ceiling and shielded by a square piece of white flannel. Thus, during the rearing period, the visual field of the animals consisted of the cage, the compartment walls and ceiling, the water bottle, the cagemates, and the stimuli for the preexposure groups.

Stimulus placement procedures were introduced at age 50 days. For the groups that received early experience with forms, the stimuli consisted of two circles, 3 in. in diam, and two equilateral triangles, $3-1 / 2$ in. per side. These shapes were constructed from 1/8-in.-thick black Plexiglas. The stimuli were suspended from the cage ceiling by means of a wire and placed so that the subjects could obtain both visual and tactual-kinesthetic feedback from the forms. There was one cutout to each wall, and their positions were rotated weekly around the cage so that their spatial proximity to food and water could be controlled.

\section{Discrimination Training}

The testing apparatus was a Grice discrimination box as modified by Gibson and Walk (1956). At the age of 110 days, the animals were given 4 days of pretraining identical to the methods outlined by Gibson and Walk (1956). Pretraining consisted of training the animals to push open the panels to obtain a food reward in the absence of visual forms attached to the panels.

After pretraining, discrimination training was begun following procedures described by Bennett and Ellis (1968) and Gibson and Walk (1956). The subject could obtain a pinch of wet mash from one of the two stimulus panels, a black circle or a black triangle on a white background, by pushing open the correct stimulus door. Half the subjects were trained to circle positive and half to triangle positive. The side of presentation of the positive stimulus was derived from Fellow's (1967) chance stimulus sequences for discrimination tasks. The criterion for task mastery was 18 out of 20 correct responses with the last 10 consecutive responses correct. The animals were run until this criterion was achieved or until they had been run on the discrimination task for a maximum of 300 trials. The rats were administered 12 trials per day.

\section{Surgery and Histology}

Following anesthetization via sodium pentobarbital $(65 \mathrm{mg} / \mathrm{kg}$ body weight) preceded by $0.1 \mathrm{cc}(0.2 \mathrm{mg})$ atropine sulfate to reduce respiratory distress, neocortical and hippocampal ablations were accomplished via aspiration (Isaacson, Douglas, \& Moore, 1961). For the hippocampal ablations, the neocortical tissue covering the hippocampus was aspirated to expose the hippocampus, which was then removed as completely as possible. Care was taken to avoid injury to the underlying thalamus. The cortical-lesion groups had the hippocampus exposed but not removed. For these latter animals, the extent of the cortical ablation was increased in an attempt to equate the total amount of brain tissue removed for both hippocampal and cortically lesioned subjects. Following completion of surgery, each rat received $0.1 \mathrm{cc}$ Mikedimide $(0.1 \mathrm{mg})$, a barbiturate antagonist, intraperitoneally. Tetracycline was added to the animal's water supply for several days following surgery as a prophylactic against infection.

Following completion of behavior testing, the lesioned animals were killed by a lethal dose of sodium pentobarbital and 

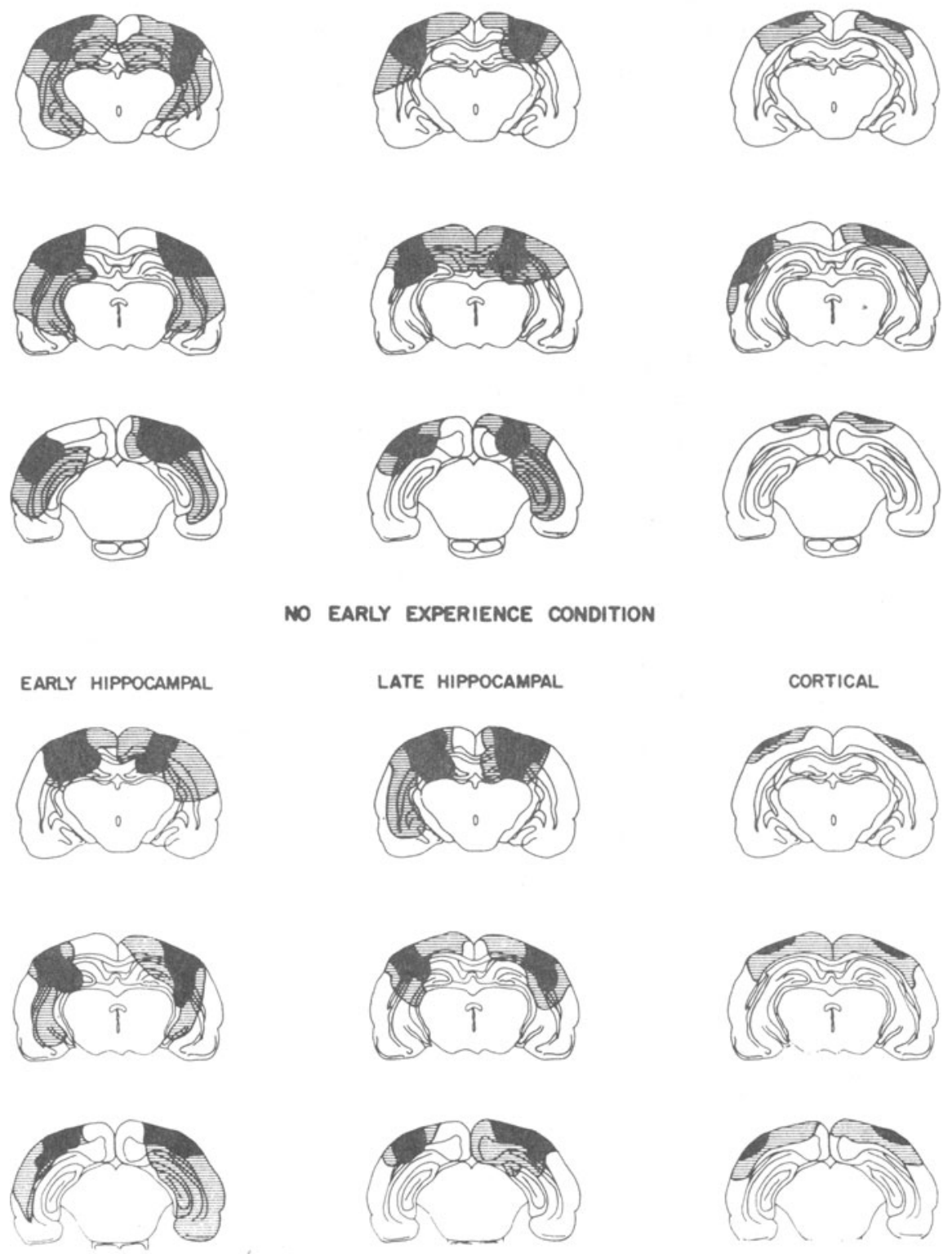

Fig. 1. Maximal and minimal extent of lesion for surgically treated animals. Black areas denote minimal and lined areas indicate maximal extent of lesion.

were intracardially perfused with saline, followed by $10 \%$ formalin. Paraffin sections were cut at 15 microns and cresyl violet stained. Sections were mounted, examined microscopically, and then reconstructed on diagrams of the rat brain taken from the Pellegrino and Cushman (1967) atlas. The posterior neocortex, corpus callosum, fimbria, and the hippocampus received damage. Examination of the brains for the hippocampal groups revealed extensive bilateral hippocampal damage estimated to average $65 \%-70 \%$. The cortical control animals generally had less total loss of brain tissue than did the hippocampal groups. In both the hippocampal and cortical control animals, the neopallial lesions involved the projection areas of the auditory and visual systems. The extent of the smallest and largest ablations for the cortical and hippocampal groups is illustrated in Fig. 1.

\section{RESULTS}

The basic data obtained in the discrimination learning task are shown in Table 1. A Kruskal-Wallis one-way analysis of variance by ranks indicated a significant difference between the groups in terms of mean trials to 
Table 1

Means, Medians, and Standard Deviations of the Performance Measures for the Eight Treatment Conditions

\begin{tabular}{lrcc}
\hline & \multicolumn{3}{c}{ Number of Trials to Criterion } \\
\cline { 2 - 4 } Condition & Mean & Median & SD \\
\hline NE & 69 & 68 & 19 \\
NNE & 108 & 108 & 34 \\
CE & 60 & 60 & 21 \\
CNE & 99 & 97 & 20 \\
EHE & 72 & 49 & 39 \\
EHNE & 104 & 103 & 32 \\
LHE & 87 & 78 & 31 \\
LHNE & 118 & 114 & 14 \\
\hline
\end{tabular}

criterion on the triangle-circle discrimination task $[\mathrm{H}(7)=25.78 ; \mathrm{p}<.001]$. Kruskal-Wallis analyses were also applied within the experience and no experience conditions. As can be seen by inspection of Table 2, no significant differences emerged within the early experience groups $[H(3)=2.54 ; p>.05]$. Thus, the performance of the groups that were preexposed to the to-be-discriminated stimuli was roughly equivalent, as was the performance of the groups not previously exposed to the to-be-discriminated stimuli, regardless of their surgical treatment. To isolate the significant source of variance between the early-experience and the no-early-experience conditions, Mann-Whitney U tests (two-tailed) were applied to the trials-to-criterion data for each surgical condition. The results of these analyses are presented in Table 3.

Examination of this table indicates that Groups NE and CE learned the task in significantly fewer trials than did animals from any group not preexposed to the to-be-discriminated shapes. Thus, early experience with forms significantly increased their later discriminability for animals in the NE and CE conditions. Of particular interest for the present study are data obtained from animals that sustained hippocampal damage. For Group EHE, experience with the to-be-discriminated stimuli did not facilitate performance as compared to normal, cortical, or early hippocampectomized subjects who were reared in the no-stimulus preexposure environment. Group EHE did master the task in significantly fewer trials than did Group LHNE. However, this difference might possibly reflect a lack of recovery from surgical trauma in the later group.

The effect of hippocampal lesions on the early-experience transfer phenomenon was further assessed by examining the rate of task acquisition of Group LHE as compared to subjects in the other early-experience conditions. This analysis was conducted to evaluate whether the effect of the lesions was to interfere with information processing and memory consolidation in the early environment rather than being due to a lack of ability to transfer this information to the novel learning situation. Analysis of these data indicated no difference between Group LHE and
Table 2

Summary of Mann-Whitney U Tests Analyzing Trials to Criterion Within Early Experience and No Early Experience Preexposure Conditions

\begin{tabular}{lcl}
\hline Group & df & U \\
\hline NE vs. CE & 8,7 & 18 \\
NE vs. EHE & 8,9 & 33 \\
NE vs. LHE & 8,7 & 16 \\
CE vs. EHE & 7,9 & 28 \\
CE vs. LHE & 7,7 & 10.5 \\
EHE vs. LHE & 9,7 & 23 \\
NNE vs. CNE & 8,7 & 35.5 \\
NNE vs. EHNE & 8,8 & 35.5 \\
NNE vs. LHNE & 8,8 & 28 \\
CNE vs. EHNE & 7,8 & 27 \\
CNE vs. LHNE & 7,8 & 20 \\
EHNE vs. LHNE & 8,8 & 20.5 \\
\hline
\end{tabular}

Note-In no case was $p$ significant.

Group NE $\quad(\mathrm{U}=16, \quad \mathrm{df}=8 / 7, \quad \mathrm{p}>.05), \quad$ Group CE $(\mathrm{U}=10.5, \mathrm{df}=7 / 7, \mathrm{p}>.05)$, or Group EHE $(\mathrm{U}=23$, $\mathrm{df}=9 / 7, \mathrm{p}>.05)$. Group LHE was also compared to animals that received no preexposure to the to-be-discriminated forms. As can be seen in Table 3, no significant differences were found between Group LHE and any of the no-early-experience control groups except for Group LHNE $(\mathrm{U}=8, \mathrm{df}=7 / 8, \mathrm{p}<.02)$; Group LHE was superior to this group. This latter observed difference suggests that the relative performance of Group LHE to the other early-experience groups and to animals in the no-early-experience conditions can possibly be accounted for by a lack of recovery from the surgical trauma occurring 5 days prior to the onset of training rather than to an inability to transfer information from

Table 3

Summary of Mann-Whitney U Tests Analyzing Trials to Criterion Between Early Experience and No Early Experience Preexposure Conditions*

\begin{tabular}{lccl}
\hline Group & df & $\mathrm{U}$ & $\mathrm{p}$ \\
\hline NE vs. NNE & 8,8 & 10.5 & .03 \\
NE vs. CNE & 8,7 & 8,5 & .03 \\
NE vs. EHNE & 8,8 & 7 & .01 \\
NE vs. LHNE & 8,8 & 1 & .001 \\
CE vs. NNE & 7,8 & 6 & .01 \\
CE vs. CNE & 7,7 & 2 & .002 \\
CE vs. EHNE & 7,8 & 6 & .01 \\
CE vs. LHNE & 7,8 & 0 & .001 \\
EHE vs. NNE & 9,8 & 17 & n.s. \\
EHE vs. CNE & 9,7 & 14.5 & n.s. \\
EHE vs. EHNE & 9,8 & 17.5 & n.s. \\
EHE vs. LHNE & 9,8 & 8 & .02 \\
LHE vs. NNE & 7,8 & 17.5 & n.s. \\
LHE vs. CNE & 7,7 & 12 & n.s. \\
LHE vs. EHNE & 7,8 & 15.5 & n.s. \\
LHE vs. LHNE & 7,8 & 8 & .02 \\
\hline
\end{tabular}

*Where comparisons are significant, group on left is superior. 
the early-experience environment to the discrimination learning situation.

\section{DISCUSSION}

The principal finding of this study was the lack of facilitation produced by early experience with the to-be-discriminated forms on later stimulus discrimination learning for animals which had their hippocampi destroyed prior to preexposure. This finding provides at least some support for the recent memory hypothesis of hippocampal functioning, a view posited on the basis of the extreme memory deficits which have been noted following hippocampectomy in humans.

On the other hand, the early-experience effect did occur for rats that had their hippocampi removed following preexposure to the shapes for 55 days when the learning rate of these subjects was compared to nonpreexposed animals which had been subjected to similar surgical treatment. This latter finding is interesting in that it points out another similarity between the present findings and the human hippocampal syndrome. Although the ability to consolidate new information is severely disrupted in human hippocampal patients, the ability to recall events which occurred prior to surgery is generally intact (Milner, 1966). The data from the late hippocampal animals do somewhat confound the study in that their lesion-testing interval was different from that experienced by the early hippocampectomized subjects; nevertheless, the late lesion data do provide some additional support for the recent memory hypothesis.

While analysis of the findings demonstrated no difference between Groups EHE and EHNE, thus implicating the hippocampus in the mediation of information processing and memory consolidation, the generality of this finding must be restricted in light of other data. Intragroup comparisons of animals that received experience with the to-be-discriminated forms indicated that Groups NE, CE, EHE, and LHE were equivalent. If the recent memory hypothesis is to account for memory consolidation of environmental stimuli, then Groups NE, CE, and LHE should have performed significantly better than Group EHE. This expectation was not borne out by the present analysis. Moreover, a recent memory account would predict equivalence between Group EHE and all no-early-experience groups. However, performance of Group EHE was significantly better than that of one of the no-early-experience groups, Group LHNE. The fact that Group EHE was superior to Group LHNE can possibly be accounted for by lack of recovery from the surgical trauma occurring 5 days prior to the onset of training for the late hippocampal animals. Nonetheless, the fact that Group EHE performed as well as Groups $\mathrm{NE}$ and CE does restrict the support of the present findings for the recent memory hypothesis.

Related research suggests two possible bases for the observed deficits in the ability to profit from preexposure to the to-be-discriminated stimuli among the early hippocampectomized animals. First, hippocampectomy may have resulted in decreased attention to the cage stimuli. This seems plausible in light of animal lesion (e.g., Jackson \& Strong, 1965; Kimble \& Kimble, 1970) and animal EEG (e.g., Bennett, 1971; Bennett, Hebert, \& Moss, 1973) findings which have implicated the hippocampus in mediating processes of attention to environmental cues. A decrease in attention to the preexposed shapes would have accounted for the lack of facilitation from early experience observed in the hippocampectomized animals.

An alternative interpretation centers on the nature of stimulus feedback required to produce the early-experience effect. It has been shown that for the albino rat, the subject used in the present inquiry, successful transfer from early experience is at least partially dependent on tactual-kinesthetic feedback from the forms. Bennett and Ellis (1968) found that albino rats which were allowed to manipulate the visually preexposed shapes learned the subsequent visual discrimination task in significantly fewer trials than those not allowed to manipulate the stimuli. These results suggested that tactual-kinesthetic feedback might aid in bringing about an ordering of the visual cues. Rickert and Bennett (1972) and Rickert, Bennett, Anderson, Corbett, and Smith (1973) have proposed that hippocampal ablations produce an animal that is deficient in the ability to consolidate information derived from response-produced feedback. If one can equate processing proprioceptive feedback during performance with the ability to process information derived from tactual-kinesthetic feedback, then a possible explanation for the observed deficits obtained in the current research emerges; namely, the hippocampus may be necessary for albino rats to process tactual-kinesthetic feedback from the preexposed visual stimuli. This information is necessary to organize stimulation derived from the visual cues. It should be pointed out that these two interpretations are not mutually exclusive.

This second account could be tested by examining the effects of early hippocampal ablations on transfer of perceptual learning for animals that do not need tactual-kinesthetic feedback for maximum transfer of early experience. Either hooded rats or hamsters could be used for such an inquiry. Both of these species have a pigmented iris and superior visual acuity to the albino rat. Neither of these species require tactual-kinesthetic feedback from the early-experience shapes to maximally profit from preexposures to visual forms (Anton \& Bennett, 1972; Bennett, Rickert, \& McAllister, 1970).

\section{REFERENCES}

Anton, B. S., \& Bennett, T. L. Role of tactual-kinesthetic feedback in transfer of perceptual learning in the golden 
hamster, Psychonomic Science, 1972, 28, 157-158.

Bennett, T. L. Hippocampal theta activity and behavior-A review. Communications in Behavioral Biology, 1971, 6 37-48.

Bennett, T. L., Anton, B. S., \& Levitt, L. Stimulus relevancy and transfer of perceptual learning. Psychonomic Science, 1971, 25, 159-160.

Bennett, T. L., \& Ellis, H. C. Tactual-kinesthetic feedback from manipulation of visual forms and nondifferential reinforcement in transfer of perceptual learning. Journal of Experimental Psychology, 1968, 77, 495-500.

Bennett, T. L., Hébert, P. N., \& Moss, D. E. Hippocampal theta activity and the attention component of discrimination learning. Behavioral Biology, 1973, 8, 173-181.

Bennett, T. L., Rickert, E. J., \& McAllister, L. E. Role of tactual-kinesthetic feedback in transfer of perceptual learning for rats with pigmented irises. Perceptual and Motor Skills, $1970,30,916-918$.

Douglas, R. J. The hippocampus and behavior. Psychological Bulletin, 1967, 67, 87-92.

Fellows, B. J. Chance stimulus sequences for discrimination tasks. Psychological Bulletin, 1967, 67, 87-92.

Gibson, E. J., \& Walk, R. D. The effect of prolonged exposure to visually presented patterns on learning to discriminate them Journal of Comparative and Physiological Psychology, 1956 , 49, 239-242.

Isaacson, R. L., Douglas, R. J., \& Moore, R. Y. The effect of radical hippocampal ablation on acquisition of avoidance response. Journal of Comparative and Physiological
Psychology, 1961, 54, 625-628.

Jackson, W. J., \& Strong, P. N., Jr. Differential effects of hippocampal lesions upon sequential tasks and maze learning by the rat. Journal of Comparative and Physiological Psychology, 1969, 68, 442-450.

Kimble, D. P. Hippocampus and internal inhibition. Psychological Bulletin, 1968, 70, 285-295.

Kimble, D. P., \& Kimble, R. J. The effect of hippocampal lesions on extinction and "hypothesis" behavior in rats. Physiology and Behavior, 1970, 5, 735-738.

Milner, B. Amnesia following operation on the temporal lobes. In C. W. M. Whitty and O. L. Zangwill (Eds.), Amnesia. London: Butterworths, 1966. Pp. 109-133.

Pellegrino, L. J., \& Cushman, A. J. A stereotaxic atlas of the rat brain. New York: Appleton-Century-Crofts, 1967.

Penfield, W., \& Milner, B. Memory deficit produced by bilateral lesions in the hippocampal zone. A. M. A. Archives of Neurology and Psychiatry, 1958, 79, 475-497.

Rickert, E. J., \& Bennet,, T. L. Performance of hippocampectomized rats on discontinuously negatively correlated reward. Behavioral Biology, 1972, 7, 395-582.

Rickert, E. J. Bennett, T. L. Anderson, G. J., Corbett, J., \& Smith, L. Differential performance of hippocampally ablated rats on nondiscriminated and discriminated DRL schedules. Behavioral Biology, 1973, 8, 597-609.

(Received for publication April 11, 1974; revision received August 5, 1974.) 